\title{
EFEITO DO FATOR XIII DA COAGULAÇÃO NA CICATRIZAÇÃO DA PELE DE RATOS EM USO DE CORTICOSTERÓIDE
}

\author{
EFFECT OF FACTOR XIII ON THE HEALING OF RAT SKIN IN USE OF \\ CORTICOSTEROID
}

\author{
Aldo da Cunha Medeiros TCBC-RN ${ }^{1}$ \\ Anísio Virgolino da Silva TCBC-RN ${ }^{2}$ \\ Francisco Pignataro Lima ${ }^{3}$ \\ Carolina Vannucci Vasconcelos ${ }^{4}$ \\ Lidiane Maria de Brito Macedo ${ }^{4}$ \\ Nara Medeiros Cunha Melo ${ }^{4}$
}

\begin{abstract}
RESUMO: Objetivos: Observar o efeito do fator XIII da coagulação (Fibrogamin ${ }^{\circledR}$ ) na cicatrização de feridas incisas da pele de ratos tratados com corticosteróide. Foi feita a avaliação quanto ao aspecto histopatológico dos tecidos em cicatrização e sua resistência à tensão. Método: Foram utilizados 40 ratos Wistar, divididos em quatro grupos. No grupo A $(n=10)$, foi administrado corticosteróide. No grupo B $(n=10)$ foi usado corticosteróide e fator XIII. No grupo $\mathrm{C}(\mathrm{n}=10)$ foi injetado fator XIII e no grupo $\mathrm{D}(\mathrm{n}=10)$ foi administrado placebo (controle). A resistência à tensão foi medida através de tensiômetro computadorizado e as alterações histopatológicas quantificadas por análise digital. Resultados: Ocorreu uma significativa diminuição da resistência da ferida de pele no grupo A (523,6gf), quando comparado com o controle (1480,4gf). No grupo B (868,8gf) notou-se significativa diferença em relação ao grupo $A(p<0,0001)$. O grupo $C$ não mostrou diferença $(p=0,067)$ em relação ao grupo controle (D), entretanto foram observadas diferenças significativas quando comparados os grupos A e C; A e D $(\mathrm{p}<0,0001)$. A análise da densidade do colágeno e de células inflamatórias revelou as mesmas diferenças observadas na resistência à tensão. Conclusões: Foi observado que a ação do corticosteróide dificultou a cicatrização da pele de ratos e diminuiu a resistência à tensão, ação revertida pelo uso do fator XIII . A utilização do fator XIII sem uso de corticosteróide não demonstrou ação de melhora nos resultados da cicatrização em relação ao controle.
\end{abstract}

Descritores: Fator XIII; Cicatrização; Pele; Corticosteróide; Resistência à tensão; Histopatologia.

\section{INTRODUÇÃO}

Estudos recentes observaram que o fator XIII da coagulação desempenha importante papel na hemostasia e na cicatrização das feridas ${ }^{1-5}$. Ele estabiliza a fibrina, estimula a proliferação de fibroblastos e sua adesão aos filamentos de fibrina, e promove a migração dessas células no coágulo de fibrina. Os corticosteróides, sabidamente, deprimem o processo de cicatrização e consequentemente a consolidação das feridas suturadas de pele e de outros tecidos ${ }^{6-8}$.
É freqüente o uso prolongado de corticosteróides por pacientes portadores de doenças crônicas, alérgicas ou inflamatórias. Quando esses pacientes apresentam concomitantemente doença com indicação cirúrgica, passam a apresentar alto risco de complicações nas feridas operatórias $^{6-8}$.

O fator XIII da coagulação é uma protransglutaminase que se torna ativada pela ação da trombina e do cálcio na fase final da cascata da coagulação. Além da presença no plasma, o fator XIII também existe nas plaquetas, monócitos e macrófagos derivados dos monócitos ${ }^{9}$. As

1. Doutor em Cirurgia; Coordenador da Disciplina de Técnica Operatória — UFRN; Chefe do Núcleo de Cirurgia Experimental — UFRN; Pesquisador nível I do CNPq.

2. Prof. Adjunto do Departamento de Cirurgia da UFRN; Mestrando do PPGCSA.

3. Prof. Assistente do Departamento de Patologia da UFRN.

4. Alunas bolsistas de iniciação científica - CNPq-PIBIC.

Recebido em 07/11/2001

Aceito para publicação em 26/03/2002

Trabalho realizado no Núcleo de Cirurgia Experimental — UFRN, apoiado pelo CNPq. 
células que se originam na medula óssea parecem ser o local primário para a síntese da subunidade $\mathrm{A}$ do fator XIII, mas o fígado também contribui para essa síntese. A subunidade B do fator XIII é sintetizada no fígado ${ }^{10,11}$. Ultimamente tem sido estudada a participação do referido fator na cicatrização das feridas, tanto em casos de deficiências do mesmo por doenças hematológicas quanto como suplementação, em animais e indivíduos normais. Tem sido observado que o fator XIII estimula a migração de fibroblastos para os coágulos de fibrina estabilizado e promove a sua proliferação ${ }^{12}$. Experimentos in vitro mostram que o crescimento de culturas de fibroblastos no plasma deficiente de fator XIII é tanto qualitativa quanto quantitativamente defeituoso. A proliferação normal de fibroblastos pode ser restaurada através da adição de fator XIII purificado. Essas e outras observações têm mostrado a importância do fator XIII na cicatrização das feridas ${ }^{3,13,14}$.

A cortisona tem efeito deletério sobre a cicatrização, por reduzir as respostas inflamatórias no início da cicatrização e por inibir a síntese dos fatores de crescimento pelos macrófagos da ferida. A inibição da liberação da histamina, a redução da migração celular e da neoangiogênese estão entre as etapas da cicatrização inibidas pelos corticosteróides. Questiona-se também a ação dessas drogas sobre a redução da beta-glicuronidase durante a fase inicial da cicatrização. Observou-se, em cães, que a administração de dexametasona causou retardo na cicatrização e a redução da resistência à tensão dos tecidos envolvidos ${ }^{6-8}$.

O presente estudo levou em consideração esses aspectos e visou observar, em laboratório, o efeito do fator XIII da coagulação (Fibrogamin ${ }^{\circledR}$ ) no processo de cicatrização da pele de ratos, previamente tratados ou não com corticosteróide. Foi feita a avaliação quanto à histopatologia dos tecidos em cicatrização e sua resistência à tensão após período de observação preestabelecido.

\section{MÉTODO}

Foram utilizados 40 ratos machos Wistar, pesando $245 \pm 15 \mathrm{~g}$, divididos aleatoriamente em quatro grupos. Os animais foram observados em gaiolas individuais com água e alimento ad libitum, anestesiados com pentobarbital sódico na dose de $20 \mathrm{mg} / \mathrm{kg}$ por via intraperitoneal, e operados sob condições assépticas. Os animais dos grupos A $(\mathrm{n}=10)$ e $\mathrm{B}(\mathrm{n}=10)$ receberam dose $\mathrm{de} 1 \mathrm{mg} / \mathrm{kg}$ intramuscular de acetato de metilprednisolona três dias antes do procedimento cirúrgico, no dia da operação e no quinto dia pós-operatório. O grupo B $(n=10)$ foi tratado com a mesma dose de metilprednisolona e 120 unidades de fator XIII da coagulação (Fibrogamin ${ }^{\circledR}$ ) IV no dia da operação e no quito dia pós-operatório. $\mathrm{O}$ grupo $\mathrm{C}(\mathrm{n}=10)$ recebeu a mesma dose de fator XIII e 1ml de solução salina IM. Os dez ratos do grupo D receberam $1 \mathrm{ml}$ de solução salina IM. Todos os animais foram observados durante 10 dias após o procedimento operatório.

Após 12 horas de jejum, ingerindo apenas água, os animais foram submetidos a anestesia com pentobarbital sódico $20 \mathrm{mg} / \mathrm{kg}$, depilação da pele do dorso, anti-sepsia com PVPI, e incisão longitudinal de $4 \mathrm{~cm}$ na pele, logo após suturada com fio de náilon 5-0. No 10ํ dia de pós-operatório cada grupo de 10 animais foi tratado com dose letal de anestésico e feita a ressecção da pele em formato de ampulheta contendo a cicatriz de permeio, de modo que no local da cicatriz se mantivesse uma largura de $2 \mathrm{~cm}$. Retirados os pontos de náilon, o tecido em cicatrização foi submetido a teste de resistência à tensão através da Máquina de Ensaios (EMICÒ) modelo DL 500 MF dotada de programa específico versão 3.0 para windows. Foi utilizada a unidade grama força (gf) para determinar a resistência à tensão. Em seguida a zona de pele em cicatrização foi fixada em formol $10 \%$ e levada para exame histopatológico através das colorações HE e tricrômico de Masson. A análise quantitativa foi feita utilizando-se um sistema digitalizador e analisador de imagens. A área total dos campos microscópicos foi observada utilizando-se microscópio óptico (Olimpus), cuja imagem foi capturada por câmera e digitalizada através do Software Image Pro-plus, versão 3.0 (Media Cybernetics - LP, USA). O campo digitalizado foi dividido pelo programa em unidades de imagem denominadas pixels, com coordenadas definidas. Dez campos aleatórios de cada lâmina foram selecionados, os impulsos ópticos digitalizados, resultando em uma imagem de cada campo que foi armazenada e processada em sistema multimídia. As lâminas foram examinadas para quantificação de densidade de colágeno e dos elementos da reação inflamatória.

A comparação entre os grupos pareados foi feita usando a análise de variância e o teste de Student, considerando-se significância de 0,05.

\section{RESULTADOS}

Os dados coletados mostraram diminuição da resistência da pele à tensão no grupo de ratos que utilizaram apenas o corticóide (grupo A), em relação aos demais grupos ( $\mathrm{p}<0,0001)$. Quando foi comparado o grupo A com o grupo B, que usou corticóide e fator XIII da coagulação, observou-se uma significativa melhora da resistência à tensão da pele suturada $(\mathrm{p}<0,0001)$, dando a idéia de que esse fator conseguiu minimizar os efeitos deletérios do corticóide sobre o processo de cicatrização da pele. A resistência à tensão nos animais do grupo $\mathrm{C}$, que usaram apenas fator XIII, não teve diferença $(\mathrm{p}=0,067)$ em relação ao grupo de controle (D). Entretanto, quando foram comparados aos pares os grupos A e C, bem como A e D, a diferença revelou-se significativa $(\mathrm{p}<0,0001)$ (Tabela 1$)$.

No que diz respeito ao estudo histopatológico, verificou-se uma nítida correlação com os achados na resistência à tensão, ou seja, o grupo A (corticóide) teve a menor densidade do colágeno e presença de fibroblastos, dados que foram melhorados pelo uso do fator XIII. No grupo B (corticóide + fator XIII) a densidade do colágeno revelou-se significativamente maior $(\mathrm{p}<0,0001)$ que no grupo A (Tabela 2). Comparando-se os grupos A e B com o controle, observou-se uma diferença significativa entre as médias dos dados histopatológicos. A administração do fator XIII nos animais do grupo C não melhorou a densida- 
de dos parâmetros histopatológicos em relação ao grupo de controle $(\mathrm{p}=0,21)$ (Tabela 2$)$. Na Figura 1 pode-se observar o aspecto da intensa deposição de colágeno em animal do grupo B e na Figura 2 o mesmo parâmetro corado em amarelo pelo programa digital, para efeito de quantificação da densidade do colágeno.

\section{DISCUSSÃO}

O propósito deste trabalho foi avaliar o efeito do fator XIII da coagulação na cicatrização da pele de ratos tratados com metilprednisolona, no que diz respeito à re-

Tabela 1

Resistência à tensão da pele de ratos tratados com corticóide e fator XIII da coagulação (observação por 10 dias)

\begin{tabular}{c|c|c|c|c}
\hline Animal $n^{\circ}$ & $\begin{array}{c}\text { Grupo } A \\
\text { (Corticóide) } g f\end{array}$ & $\begin{array}{c}\text { Grupo B } \\
\text { (Corticóide }+ \text { Fator XIII) } g f\end{array}$ & $\begin{array}{c}\text { Grupo C } \\
\text { (Fator XIII) gf }\end{array}$ & $\begin{array}{c}\text { Grupo D } \\
\text { (Controle) } g f\end{array}$ \\
\hline 1 & 554 & 780 & 1435 & 1304 \\
2 & 424 & 900 & 1320 & 1278 \\
3 & 646 & 876 & 1649 & 1615 \\
4 & 523 & 798 & 1703 & 1470 \\
5 & 570 & 874 & 1736 & 1770 \\
6 & 490 & 1056 & 1790 & 1789 \\
7 & 486 & 896 & 1350 & 1331 \\
8 & 588 & 890 & 1420 & 1272 \\
10 & 475 & 879 & 1575 & 1600 \\
Média & 480 & 739 & 1849 & 1375 \\
Desvio Padrão & 523,6 & 868,8 & 1582,7 & 1480,40 \\
\hline
\end{tabular}

Teste " $t$ " para grupos pareados:

Comparação entre A e B: $t$ calculado $=-9,5 ; p<0,0001$

A e $C:$ t calculado $=-17,4 ; p<0,0001$

A e D: t calculado $=-15,36 ; p<0,0001$

$B$ e C: $t$ calculado $=-10,5 ; p<0,0001$

$B$ e D: $t$ calculado $=-11,08 ; p<0,0001$

C e D: $t$ calculado $=2,07 ; p=0,067$ (não significante)

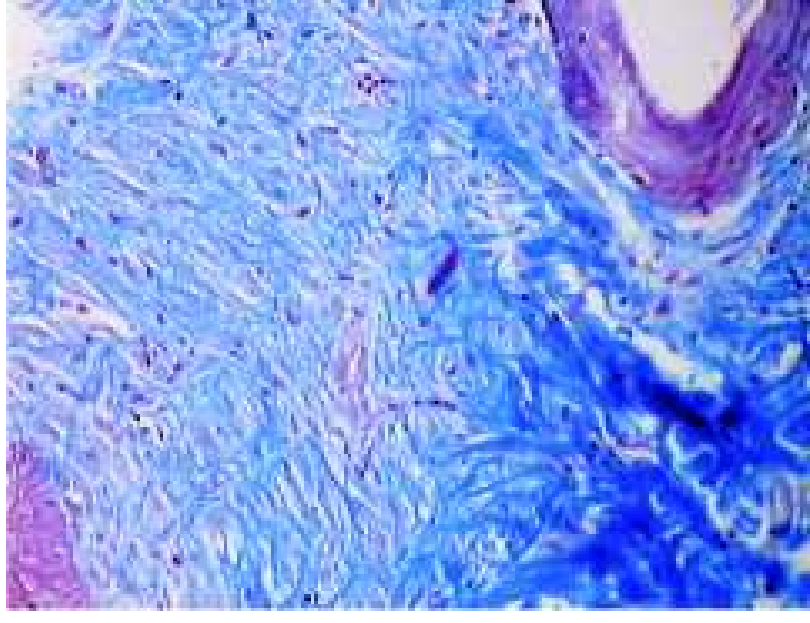

Figura 1 - Observa-se a intensa densidade do colágeno corado em azul pelo tricrômico de Masson em animal do grupo $B$. Nos animais do grupo A, em uso de corticosteróide, observouse densidade menor em todas as lâminas examinadas (200X).

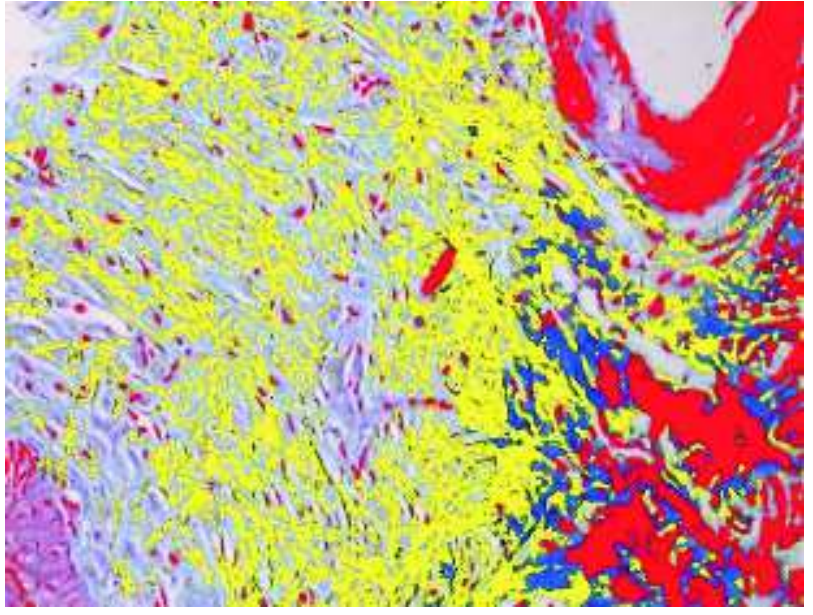

Figura 2 - As fibras colágenas, que correspondem à Figura 1, encontram-se coradas em amarelo, para efeito de quantificação através do programa Image Pro-plus (200 X). 
Tabela 2

Densidade do colágeno e de fibroblastos no tecido em cicatrização da pele de ratos tratados com corticóide e fator XIII da coagulação, comparados com o grupo de controle

\begin{tabular}{ccccc}
\hline & $\begin{array}{c}\text { Grupo A } \\
\text { Animal } n^{\circ}\end{array}$ & $\begin{array}{c}\text { Grupo B } \\
\text { (Corticóide) } g f\end{array}$ & $\begin{array}{c}\text { Grupo C } \\
\text { (Corticóide }+ \text { Fator XIII) } g f\end{array}$ & $\begin{array}{c}\text { Grupo D } \\
\text { (Controle) } g f\end{array}$ \\
\hline 1 & 5600,30 & 10876,12 & 20321,00 & 35786,00 \\
2 & 4326,98 & 9356,30 & 17453,00 & 13769,50 \\
3 & 6289,20 & 12765,00 & 19651,65 & 20289,60 \\
4 & 4200,90 & 8458,90 & 16378,20 & 15367,07 \\
5 & 4578,70 & 10456,20 & 21098,80 & 12785,04 \\
6 & 2200,87 & 12543,80 & 25256,87 & 14078,54 \\
7 & 3400,75 & 11764,78 & 22035,22 & 20986,20 \\
8 & 3576,45 & 10369,76 & 25894,12 & 18345,98 \\
9 & 4008,34 & 11989,23 & 28908,38 & 21763,87 \\
10 & 4876,00 & 9321,45 & 30217,45 & 22387,90 \\
Média & 3834,57 & 10790,45 & 22721,47 & 19555,5 \\
D. Padrão & 887,55 & 1464,63 & 4681,47 & 6726,72 \\
\hline
\end{tabular}

Teste " $t$ ” para grupos pareados:

Comparação entre A e B: t calculado $=-10,5 ; p<0,0001$

A e $C:$ t calculado $=-11,3 ; p<0,0001$

A e D: $t$ calculado $=-7,69 ; p<0,001$

$B$ e $C: t$ calculado $=-8,30 ; p<0,001$

$B$ e D: $t$ calculado $=-4,16 ; p=0,002$

C e D: t calculado $=1,31 ; p=0,21$ (Não significante)

sistência da ferida à tensão e sua evolução do ponto de vista histopatológico. Estudo de laboratório revelou que o fator XIII da coagulação estimula a migração de fibroblastos para um coágulo de fibrina estabilizado e promove a sua proliferação ${ }^{12}$. Experimentos in vitro mostram que o crescimento das culturas de fibroblastos no plasma de deficientes de fator XIII é tanto quantitativa quanto qualitativamente defeituoso. A proliferação normal de fibroblastos pode ser restaurada através da adição de fator XIII purificado, observações essas que estão em concordância com a importância da droga na cicatrização das feridas $^{1,15}$. No presente trabalho observou-se proliferação de tecido fibroso na pele em cicatrização, com aumento significativo de fibroblastos nos grupos de animais que fizeram uso de fator XIII.

Alguns estudos têm observado os efeitos do fator XIII na cicatrização de lesões. D'Argenio e cols. ${ }^{1}$ relataram cicatrização em menor tempo de lesões gástricas de estresse. Foi relatado o uso tópico do fator XIII em escaras de decúbito de pacientes vítimas de lesões de medula espinhal. Os autores concluíram que houve um efeito positivo no tratamento das lesões, sugerindo que o seu uso oferece ao cirurgião uma ferramenta adicional em casos complicados ${ }^{3}$.

Ensaio clínico realizado em pacientes portadores de úlcera crônica de perna de origem vascular demonstrou que o uso tópico de fator XIII reverteu as anormalidades fibrinolíticas e as alterações da permeabilidade vascular. Ao mesmo tempo em que foi feito o enfaixamento compressivo dos membros afetados, foi empregado o fator XIII em tratamento tópico diário, monitorando-se seus efeitos através de densitometria e enzimologia da fibrina. Os autores observaram uma significativa redução na superfície das feridas, comparando com o uso de placebo. Concluíram que o fator XIII é uma droga antifibrinolítica eficaz para o tratamento de úlceras crônicas de perna, provocando o seu fechamento mais rápido através de aplicações tópicas ${ }^{16}$.

O fator XIII tem-se mostrado de grande valia na cicatrização de vários tecidos, em várias condições em que ocorrem doenças intercorrentes. Desse modo, seu efeito foi testado como um fator estabilizador de fibrina na cicatrização de defeitos do osso em ratos normais e diabéticos. A deposição de colágeno foi evidente e apareceu mais orientada nos ratos diabéticos tratados com fator XIII, e sinais de deposição de osso iniciaram mais precocemente no grupo experimental do que no grupo de controle. Por outro lado, o fator XIII não afetou significativamente a cicatrização nos ratos não diabéticos ${ }^{17}$. No estudo aqui relatado foi observado o mesmo fenômeno, ou seja, nos animais que utilizaram corticosteróide ocorreu melhora nos parâmetros estudados quando se usou o fator XIII, de modo especial a deposição do colágeno, o mesmo não ocorrendo nos animais sem uso do corticosteróide, quando comparados com o controle. Conclusão idêntica foi descrita em estudo experimental realizado em modelo de fratura óssea em ratos, no qual foi postulado que o fator XIII deve ser indicado apenas, em casos de alto risco, associados a doenças intercorrentes ou sabidamente com níveis séricos baixos de fator XIII ${ }^{18}$. 
No tecido epitelial o fator XIII tem revelado efeito benéfico. Estudo in vitro realizado com células epiteliais do intestino revelou que o fator XIII estimulou de modo significativo a renovação das células do epitélio intestinal, e esse estímulo ocorreu 2,5 vezes maior do que no grupo controle. Observou também o estudo que os efeitos modulatórios do fator XIII não foram bloqueados pelo antifator de crescimento transformador beta. Esse achado pode explicar efeitos benéficos do fator XIII no tratamento da retocolite ulcerativa e na doença de $\mathrm{Crohn}^{5}$.

Os corticosteróides comprovadamente retardam o processo cicatricial das feridas, conforme evidências de autores que estudaram seus efeitos na cicatrização das feridas $^{19-22}$, a maioria deles revelando déficit na resistência das feridas e pior desempenho do ponto de vista histopatológico do que os grupos controle. $\mathrm{O}$ mecanismo inibitório da ação dos costicosteróides na cicatrização das feridas não está completamente elucidado. Entre outros fatores, foi demonstrada a diminuição da beta-glicuronidase durante os sete primeiros dias de cicatrização em ratos submetidos à ação de corticosteróides, com conseqüente diminuição da resistência das feridas desses animais ${ }^{23}$. No presente trabalho foi observada diminuição significativa da resistência à tensão da pele cicatrizada sob efeito da metilprednisolona, com nítido efeito retardante da evolução histopatológica do tecido em cicatrização.

Os dados coletados permitem concluir que o fator XIII da coagulação, quando usado por via venosa em ratos tratados com metilprednisolona, contribuiu para reverter os efeitos deletérios do corticosteróide na cicatrização da pele. O fator XIII, quando usado em animais não deprimidos pelo corticóide, não melhorou os parâmetros resistência à tensão e deposição de colágeno, em relação ao grupo de controle.

\begin{abstract}
Objetive: There is increasing evidence that coagulation factor XIII is protective in models of wound healing. Our purpose is to observe the effects of coagulation factor XIII on the healing of skin wound of rats treated with corticosteroid. Method: Fourty Wistar rats, weighing $245 \pm 15 \mathrm{~g}$, were randomly divided into four groups. In group $A(n=10)$ the rats received corticosteroid IM. In group $B(n=10)$ corticosteroid and factor XIII were used IM. In group $C(n=10)$ the rats received only factor XIII and in group $D(n=10)$ saline solution was used (control). The tensil strenght of the wound was measured by a computed tensiometer and the hystopatologic evolution of healing was quantitated by a digital system. Results: The results indicated a significant difference ( $p<0.0001)$ of tensil strenght between group A ((523.6gf) and the control $(D)$ group $(1480.4 \mathrm{gf})$. In group B the tensil strenght (868.8gf) was significantly greater than in group A ( $p<0.0001)$. The factor XIII didn't increase the tensil strenght in group $C$, when compared with control $(p=0,067)$. The hystopatologic analysis indicated a similar tendency observed in tensil strenght. Conclusions: There was a strong correlation between factor XIII, tensil strenght and healing of sutured skin wounds of rats treated with corticosteroid. The fator XIII didn't change the wound healing in rats with no corticosteroid.
\end{abstract}

Key Words: Factor XIII; Healing; Skin; Corticosteroid; Tensil strenght; Hystopatology.

\section{REFERÊNCIAS}

1. D'argenio G, Iovino P, Cosenza V et al. Factor XIII improves gastric stress lesions in rats. Digestion 2001; 63: 220-208.

2. Mandai R, Eguchi Y, Tanaka M, Sai Y, Nosaka S. Effect of profound hemodilution on small-intestinal wound healing in rabbits. J Surg Res 2001; 99: 107-113.

3. Becker SW, Weidt F, Rohl K. The role of plasma transglutaminase (F XIII) in wound healing of complicated pressure sores after spinal cord injury. Spinal Cord 2001; 39: 114-117.

4. Ohwada S, Sato Y, Sato N et al. Effects of transfusion on gastrointestinal anastomotic wound healing and leukocite function in rats. Eur Surg Res 2000; 32: 3530358

5. Cario E, Goebell H, Dignass AU. Factor XIII modulates intestinal epitelial wound healing in vitro. Scand J Gastroenterol 1999; 34: 485-490.

6. Montovani M, Leonardi LS, Alcântara FG. Evolução da cicatrização de anastomoses do intestino grosso em condições de normalidade e sob ação de drogas imunossupressoras.Rev Paul Med 1979;94:118-26

7. Martins Jr A, Guimarães AS, Ferreira AL. Efeito dos corticosteróides na cicatrização de anastomoses intestinais. Acta Cir Bras 1992; 7: 28-30.

8. Phillips JD, Kim CS et al. Effects of chronic corticosteroids and vitamin A on the healing of intestinal anastomosis. Am J Surg 1992; 163: 71-7.

9. Muszbek L, Yee VC, Hevessy Z. Blood coagulation factor XIII: structure and function. Tromb Res 1999; 94: 271305.

10. Egbring R, Kroniger A, Seitz D. Factor XIII deficiency: pathogenic mechanisms and clinical significance. Semin Tromb Hemost 1996; 22: 419-425.

11. Muszbek L, Adany R, Nikkola H. Novel aspects of blood coagulation factor XIII. I-Structure, distribution, activation and function. Crit Ver Clin Lab Sci 1996; 33: 357-421.

12. Ueyama M, Urayama T. The role of factor XIII in fibroblast proliferation. Jpn Exp Med 1978; 48: 135-142. 
13. Francis CHW, Marder VJ. Increased resistence to plasma degradation of fibrin with highly crosslinked alphapolymer chains formed at high factor XIII concentrations. Blood 1988; 71: 1361-1365.

14. Gerngross H, Steinmann R, Graf K. Factor XIII and bone union. A comparative clinical trials of its effect on the healing of pseudoarthroses. Med Welt 1987;38: 1533-1538.

15. Noll T, Wozniac G, McCarson K et al. Effect of factor XIII on endothelial barrier function. J Exp Med 1999; 189: 1373-1382.

16. Herouy Y, Hellstern MO, Wolfgang V et al. Factor XIIImediated inhibition of fibrinolysis and venous leg ulcers. Lancet 2000; 355: 1970-1971.

17. El-hakin EI. The effect of fibrin stabilizing factor (F.XIII) on healing of bone defects in normal and uncontroled diabetic rats. Int J Oral Maxillofac Surg 1999; 28: 304-308.

18. Hellerer O, Bruckner WL, Frey KW et al. Fracture healing under factor XIII medication. Arch Orth Trauma 1980; 97: $157-159$.

19. Clark RA. Regulation of fibroplasia in cutaneous wound repair. J Pediatr Surg 1993; 306: 42-46.

20. Makela JT, Kiviniemi H, Juvonen T et al. Factors influencing wound deiscence after midline laparotomy. Am J Surg 1995; 170: 387-389.

21. Dostal GH, Gamelli R. The differential effect of costicosteroids on wound disruption strenght in mice. Arch Surg 1990; 125: 636-638.
22. Niggebrigge AHP, Hansen BE, Trimbos JB et al. Mechanical factors influencing the incidence of burst abdomen. Eur J Surg 1995; 161: 655-658.

23. Manning JP, Dipasquale G. The influence of vitamine A and/or hidrocortisone on the beta-gluconidase activity of healing wounds in rats. Acta Physiol Pharmac Neerl 1967; 14: 460-463.

Endereço para correspondência: Dr. Aldo da Cunha Medeiros Av. Miguel Alcides Araújo, 1889 59078-270 — Natal-RN 